\title{
Ray splitting in paraxial optical cavities
}

\author{
G. Puentes, A. Aiello, J. P. Woerdman \\ Huygens Laboratory, Leiden University, P.O. Box 9504, Leiden, The Netherlands
}

November 5, 2018

\begin{abstract}
We present a numerical investigation of the ray dynamics in a paraxial optical cavity when a ray splitting mechanism is present. The cavity is a conventional two-mirror stable resonator and the ray splitting is achieved by inserting an optical beam splitter perpendicular to the cavity axis. We show that depending on the position of the beam splitter the optical resonator can become unstable and the ray dynamics displays a positive Lyapunov exponent.
\end{abstract}

PACS numbers: 42.60.Da, 42.65.Sf, 42.15.-i

\section{INTRODUCTION}

A beam splitter (BS) is an ubiquitous optical device in wave optics experiments, used e.g., for optical interference, homodyning, etc. In the context of geometrical optics, light rays are split into a transmitted and reflected ray by a BS. Ray splitting provides an useful mechanism to generate chaotic dynamics in pseudointegrable ${ }^{11}$ and soft-chaotic ${ }^{23}$ closed systems. In this paper we exploit the ray splitting properties of a BS in order to build an open paraxial cavity which shows irregular ray dynamics as opposed to the regular dynamics displayed by a paraxial cavity when the BS is absent.

Optical cavities can be classified as stable or unstable depending on the focussing properties of the elements that compose it ${ }^{[4}$ An optical cavity formed by 2 concave mirrors of radii $R$ separated by a distance $L$ is stable when $L<2 R$ and unstable otherwise. If a light ray is 
injected inside the cavity through one of the mirrors it will remain confined indefinitely inside the cavity when the configuration is stable but it will escape after a finite number of bounces when the cavity is unstable (this number depends on the degree of instability of the system). Both stable and unstable cavities have been extensively investigated since they form the basis of laser physics! Our interest is in a composite cavity which has both aspects of stability and instability. The cavity is made by two identical concave mirrors of radii $R$ separated by a distance $L$, where $L<2 R$ so that the cavity is globally stable. We then introduce a beam splitter (BS) inside the cavity, oriented perpendicular to the optical axis (Fig 1). In this way the BS defines two subcavities. The main idea is that depending on the position of the BS the left (right) subcavity becomes unstable for the reflected rays when $L_{1}\left(L_{2}\right)$ is bigger than $R$, whereas the cavity as a whole remains always stable $\left(L_{1}+L_{2}<2 R\right)$ (Fig. 2).

Our motivation to address this system originates in the nontrivial question whether there will be a balance between trapped rays and escaping rays. The trapped rays are those which bounce infinitely long in the stable part of the cavity, while the escaping ones are those which stay for a finite time, due to the presence of the unstable subcavity. If such balance exists it could eventually lead to transient chaos since it is known in literature that instability (positive Lyapunov exponents) and mixing (confinement inside the system) form the skeleton of chaos. The BS is modelled as a stochastic ray splitting element ${ }^{21}$ by assuming the reflection and transmission coefficients as random variables. Within the context of wave optics this model corresponds to the neglect of all interference phenomena inside the cavity; this would occur, for instance when one injects inside the cavity a wave packet (or cw broad band light) whose longitudinal coherence length is very much shorter than the smallest characteristic length of the cavity. The stochasticity is implemented by using a Monte Carlo method to determine whether the ray is transmitted or reflected by the $\mathrm{BS} .^{[2]}$ When a ray is incident on the ray splitting surface of the BS, it is either transmitted through it with probability $p$ or reflected with probability $1-p$, where we will assume $p=1 / 2$, i.e. we considered a $50 \% / 50 \%$ beam splitter (Fig [3). 
We then follow a ray and at each reflection we use a random number generator with a uniform distribution to randomly decide whether to reflect or transmit the incident ray.

Our system bears a close connection with the stability of a periodic guide of paraxial lenses as studied by Longhi ${ }^{6}$ While in his case a continuous stochastic variable $\epsilon_{n}$ represents a perturbation of the periodic sequence along which rays are propagated, in our case we have a discrete stochastic parameter $p_{n}$ which represents the response of the BS to an incident ray. As will be shown in section II, this stochastic parameter can take only two values, either 1 for transmitted rays or -1 for reflected ray; in this sense, our system (as displayed in Fig 4) is a surprisingly simple realization of a bimodal stochastic paraxial lens guide.

The structure of the paper is as follows. In section II we describe the ray limit, and the paraxial map or ABCD matrix associated with rays that propagate very close to the axis of the cavity. In section III we present the results of the numerical simulations for the paraxial map associated with our ray optical system; these simulations are based on standard numerical tools developed in non-linear dynamics theory. Finally, in section IV, we detail the conclusions of our work.

\section{Ray Dynamics and the Paraxial Map}

The time evolution of a laser beam inside a cavity can be approximated classically by using the ray optics limit, where the wave nature of light is neglected. Generally, in this limit the propagation of light in a uniform medium is described by rays which travel in straight lines, and which are either sharply reflected or refracted when they hit a medium with a different refractive index. To fully characterize the trajectory of a ray in a strip resonator or in a resonator with rotational symmetry around the optical axis, we choose a reference plane $z=$ constant (perpendicular to the optical axis $\hat{z}$ ), so that a ray is specified by two parameters: the height $q$ above the optical axis and the angle $\theta$ between the trajectory and the same axis. Therefore we can associate a ray of light with a two dimensional vector $\vec{r}=(q, \theta)$. This is illustrated in the 
two mirror cavity show in Fig. 3, where the reference plane has been chosen to coincide with the beam splitter (BS). Given such a reference plane $z$, which is also called Poincaré Surface of Section (SOS) ${ }^{7}$ a round trip (evolution between two successive reference planes) of the ray inside the cavity can be calculated by the monodromy matrix $M_{n}$, in other words $\vec{r}_{n+1}=M_{n} \vec{r}_{n}$, where the index $n$ determines the number of round trips. The monodromy matrix $M_{n}$ describes the linearized evolution of a ray that deviates from a reference periodic orbit. A periodic orbit is said to be stable if $\left|\operatorname{Tr} M_{n}\right|<2$. In this case nearby rays oscillate back and forth around the stable periodic orbit with bounded displacements both in $q$ and $\theta$. On the other hand when $\left|\operatorname{Tr} M_{n}\right| \geq 2$ the orbit is said to be unstable and rays that are initially near this reference orbit become more and more displaced from it.

For paraxial trajectories, where the angle of propagation relative to the axis is taken to be very small (i.e. $\sin (\theta) \cong \tan (\theta) \cong \theta$ ), the reference periodic trajectory coincides with the optical axis and the monodromy matrix is identical to the ABCD matrix of the system. The ABCD matrix or paraxial map of an optical system is the simplest model one can use to describe the discrete time evolution of a ray in the optical system. $\stackrel{4}{ }$ Perhaps the most interesting and important application of ray matrices comes in the analysis of periodic focusing (PF) systems in which the same sequence of elements is periodically repeated many times down in cascade. An optical cavity provides a simple way of recreating a PF system, since we can think of a cavity as a periodic series of lenses (see Fig 4). In the framework of geometric ray optics, PF systems are classified, as are optical cavities, as either stable or unstable.

Without essential loss of generality we restrict ourselves to the case of a symmetric cavity (i.e. two identical spherical mirrors of radius of curvature $R$ ). We take the SOS coincident with the surface of the BS. After intersecting a given reference plane $z_{i}$, a ray is transmitted (reflected), it will undergo a free propagation over a distance $L_{2}\left(L_{1}\right)$, followed by a reflection on the curved mirror $M_{2}\left(M_{1}\right)$, and continue propagating over the distance $L_{2}\left(L_{1}\right)$, to hit the surface of the 
beam splitter again at $z_{i+1}$. In Fig 4 the sequence of $z_{i}$ represents the successive reference planes after a round trip. In the paraxial approximation each round trip (time evolution between two successive intersections of a ray with the beam splitter) is represented by:

$$
\begin{aligned}
& q_{n+1}=A_{n} q_{n}+B_{n} \theta_{n}, \\
& \theta_{n+1}=C q_{n}+D_{n} \theta_{n},
\end{aligned}
$$

where

$$
\begin{gathered}
A_{n}=1-2 L_{n} / R, \quad B_{n}=2 L_{n}\left(1-L_{n} / R\right), \\
C=-2 / R, \quad D_{n}=1-2 L_{n} / R
\end{gathered}
$$

and

$$
L_{n}=\frac{L+p_{n} a}{2}
$$

We have defined $L=L_{1}+L_{2}$ and $a=L_{2}-L_{1}$; the stochastic parameter $p_{n}= \pm 1$ determines whether the ray is transmitted $\left(p_{n}=1\right)$ or is reflected $\left(p_{n}=-1\right)$.

The elements of the ABCD matrix depend on $n$ because of the stochastic response of the BS, which determines the propagation for the ray in subcavities of different length (either $L_{1}$ or $\left.L_{2}\right)$. In this way a random sequence of reflections $\left(p_{n}=1\right)$ and transmissions $\left(p_{n}=-1\right)$ represents a particular geometrical realization of a focusing system. If we want to study the evolution of a set of rays injected in the cavity with different initial conditions $\left(q_{0}, \theta_{0}\right)$, we have two possibilities, either use the same random sequence of reflections and transmissions for all rays in the set or use a different random sequence for each ray. In the latter case, we are basically doing an ensemble average over different geometrical configurations of focusing systems. As we shall see later it is convenient, for computational reasons, to adopt the second method.

In the next section we report several dynamical quantities that we have numerically calculated for paraxial rays in this system, using the map described above (Eq II) . The behavior 
of these quantities, namely, the SOSs, the exit basins, the Lyapunov exponent and the escape rate, is analyzed as a function of the displacement $(\Delta)$ of the BS with respect to the center of the cavity (see Fig 1).

\section{Results}

The paraxial map of Eq1 describes an unbounded system, that is rays are allowed to go infinitely far from the cavity axis. In order to describe a physical paraxial cavity we have to keep the phase space bounded, i.e. it is necessary to artificially introduce boundaries for the position and the angle of the ray ${ }^{[}$The phase space boundaries that we have adopted to decide whether a ray has escaped after a number of bounces or not is the beam waist $\left(w_{0}\right)$ and the diffraction half-angle $\left(\Theta_{0}\right)$ of a gaussian beam confined in a globally stable two-mirror cavity.

Measured at the center of the cavity, $w_{0}^{2}=\frac{L \lambda_{L i g h t}}{\pi} \sqrt{\frac{2 R-L}{4 L}}$ and the corresponding diffraction half-angle $\Theta_{0}=\arctan \left(\frac{\lambda_{\text {Light }}}{\pi w_{0}}\right) \stackrel{4}{4}$ For our cavity configuration we assume $R=0.15 \mathrm{~m}, L=0.2 \mathrm{~m}$ and $\lambda_{\text {Light }}=500 \mathrm{~nm}$, from which follows that $w_{0}=5.3 \times 10^{-5} \mathrm{~m}$ and $\Theta_{0}=0.15 \times 10^{-3} \mathrm{rad}$. One should keep in mind that this choice is somewhat arbitrary and other choices are certainly possible. The effect of this arbitrariness on our results will be discussed in detail in section D.

\subsection{Poincaré surface of section (SOS)}

We have first calculated the SOS for different positions of the BS. In order to get a qualitative idea of the type of motion, we have chosen as transverse phase space variables $y=q$ and $v_{y}=\sin (\theta) \approx \theta$. The successive intersections of a trajectory with initial transverse coordinates $q_{0}=1 \times 10^{-5} \mathrm{~m}, \theta_{0}=0$ are represented by the different black points in the surface of section. The different SOSs are shown in Fig [5, In Fig 5 (a) we show the SOS for $\Delta=0$, while in (b) $\Delta=1 \times 10^{-3} \mathrm{~m}$ and in (c) $\Delta=2 \times 10^{-2} \mathrm{~m}$. In (a) it is clear that the motion is completely regular (non-hyperbolic); the on-axis trajectory represents an elliptic fixed point for the map. 
In (b), where the BS is slightly displaced from the center $\left(\Delta=1 \times 10^{-3} \mathrm{~m}\right)$ we can see that this same trajectory becomes unstable because of the presence of the BS, and spreads over a finite region of the phase space to escape after a large number of bounces $\left(n=5 \times 10^{4}\right)$. In this case we may qualify the motion as azimuthally ergodic. The fact that the ray-splitting mechanism introduced by the BS produces ergodicity is a well known result ${ }^{2}$ for a closed billard. We find here an analogue phenomenon, with the difference that in our case the trajectory does not explore uniformly (but only azimuthally) the available phase space, because the system is open. Finally, in (c) we see that the fixed point in the origin becomes hyperbolic, and the initial orbit escapes after relatively few bounces $(n=165)$.

\subsection{Exit basin diagrams}

It is well known that chaotic hamiltonian systems with more than one exit channel exhibit irregular escape dynamics which can be displayed, e.g., by plotting the exit basin. ${ }^{9}$ For our open system we have calculated the exit basin diagrams for three different positions of the BS (Fig 6] $)$. These diagrams can be constructed by defining a fine grid $(2200 \times 2200)$ of initial conditions $\left(q_{0}, \theta_{0}\right)$. We then follow each ray for a sufficient number of bounces so that it escapes from the cavity. When it escapes from above $\left(\theta_{n}>0\right)$ we plot a black dot in the corresponding initial condition, whereas when it escapes from below $\left(\theta_{n}<0\right)$ we plot a white dot.

In Fig [6 (a) we show the exit basins for $\Delta=0.025 \mathrm{~m}$, the uniformly black or white regions of the plot correspond to rays which display a regular dynamics before escaping, and the dusty region represents the portion of phase space where there is sensitivity to initial conditions. In Fig. 6] (b), we show the same plot for $\Delta=0.05 \mathrm{~m}$, and in (c) for $\Delta=0.075 \mathrm{~m}$.

The exit basins plots in Fig [6 illustrate how the scattering becomes more irregular as the BS is displaced from the center. In particular, we see how regions of regular and irregular dynamics become more and more interwoven as $\Delta$ increases. Instead, for small values of $\Delta$ as in Fig 6 (a), 
we can see that there is a single dusty region with a uniform distribution of white and black dots in which no islands of regularity are present.

\subsection{Escape rate and Lyapunov exponent}

The next dynamical quantities we have calculated are the escape rate $\gamma$ and the Lyapunov exponent $\lambda$. The escape rate is a quantity that can be used to measure the degree of openness of a system ${ }^{[8}$ For hard chaotic systems (hyperbolic), the number $N_{n}$ of orbits still contained in the phase space after a long time (measured in number of bounces $n$ ) decreases as $N_{0} \exp (-\gamma n)$, while for soft chaotic systems, the stickiness to Kolmogorov-Arnold-Moser (KAM) islands (or islands of stability) leads to a power law decay $N_{0} n^{-\gamma ! 10}$ The Lyapunov exponent is the rate of exponential divergence of nearby trajectories.

Since both $\lambda$ and $\gamma$ are asymptotic quantities they should be calculated for very long times. In our system long living trajectories are rare, and in order to pick them among the grid of initial conditions $N_{0}$ one has to increase $N_{0}$ beyond the computational capability. To overcome this difficulty we choose a different random sequence for each initial condition. In this way we greatly increase the probability of picking long living orbits given by particularly stable random sequences. These long living orbits in turn make possible the calculation of asymptotic quantities such as $\lambda$ or $\gamma$.

The escape rate $\gamma$ was determined measuring $N_{n}$, as the slope of a linear fit in the $N_{n} / N_{0}$ versus $n$ curve, in a logarithmic scale; the total number of initial conditions $N_{0}$ being chosen as $2200 \times 2200$.

We have calculated the dependence of $\gamma$ with the displacement $\Delta$ of the BS from the center of the cavity, where $0 \leq \Delta \leq L / 2$. Since for $\Delta>R-L / 2$ the left subcavity becomes unstable, it would seem natural to expect that this position of the BS would correspond to a critical point. However, we have found by explicit calculation of both the Lyapunov exponent and 
the escape rate, that such a critical point does not manifest itself in a sharp way, rather we have observed a finite transition region (as opposed to a single point) in which the functional dependence of $\lambda$ and $\gamma$ change in a smooth way. In Fig 7 (a) we show the typical behavior of $\frac{N_{n}}{N_{0}} \mathrm{Vs} n$ in semi-logarithmic plot for three different positions of the BS. The displacement of the BS is $\Delta=0.0875 \mathrm{~m}, 0.05 \mathrm{~m}$ and $0.03125 \mathrm{~m}$, and the corresponding slopes (escape rate $\gamma$ measured in units of the inverse number of bounces $n$ ) of the linear fit are $\gamma=0.17693 n^{-1}$, $0.05371 n^{-1}$ and $0.01206 n^{-1}$ respectively. We have found that the decay is exponential only up to a certain time (approximately 70-1000 bounces depending on the geometry of the cavity) due the discrete nature of the grid of initial conditions.

In Fig 7 (b) we see that $\gamma$ increases with $\Delta$, revealing that for more unstable configurations there is a higher escape rate, as expected. Its also interesting to notice that the exponential decay fits better when the beam splitter is further from the center position, since this leads to smaller stability of the periodic orbits of the system. However, the dependence of the escape rate with the position of the $\mathrm{BS}$ is smooth and reveals that the only critical displacement, where the escape rate becomes positive, is $\Delta=0$.

As a next step, we have calculated the Lyapunov exponent $\lambda$ for the paraxial map; $\lambda$ is a quantity that measures the degree of stability of the reference periodic orbit. For a twodimensional hamiltonian map there are two Lyapunov exponents $\left(\lambda_{1}, \lambda_{2}\right)$ such that $\lambda_{1}+\lambda_{2}=0$. In the rest of the paper we shall indicate with $\lambda$ the positive Lyapunov exponent which quantifies the exponential sensitivity to the initial conditions. We have calculated $\lambda$ for the periodic orbit on axis, using the standard techniques, 11 and we have found that the Lyapunov exponent grows from zero with the distance of the BS to the center (Fig [7(c)). Therefore, the only critical point revealed by the ray dynamics is again the center of the cavity $(\Delta=0)$, where the magnitudes change from zero to a positive value. This result also shows that the presence of the BS with its stochastic nature introduces exponential sensitivity to initial conditions in the system for every $\Delta \neq 0$, even when both subcavities are stable. This surprising fact can be explained by taking 
into account the well known probabilistic theorem by Furstenberg on the asymptotic limit of the rate of growth of a product of random matrices (PRM) ${ }^{12}$ From this theorem we expect that the asymptotic behavior of the product $M_{n}$ of a uniform sequence $\omega$ of independent, random, unimodular, $D \times D$ matrices, and for any nonzero vector $\vec{y} \in \Re^{D}$ :

$$
\lim _{n \rightarrow \infty} \frac{1}{n}\left\langle\ln \left|M_{n} \vec{y}\right|\right\rangle=\lambda_{1}>0
$$

where $\lambda_{1}$ is the maximum Lyapunov characteristic exponent of the system, and the angular bracket indicates the average over the ensemble $\Omega$ of all possible sequences $\omega$. This means that for PRM the Lyapunov exponent is a nonrandom positive quantity. In general, it can be said that there is a subspace $\Omega^{*}$ of random sequences which has a full measure (probability 1) over the whole space of sequences $\Omega$ for which nearby trajectories deviate exponentially at a rate $\lambda_{1}$. Although there exist very improbable sequences in $\Omega$ which lead to a different asymptotic limit, they do not change the logarithmic average $(\mathrm{Eq}[2)$ [13 We have verified this result, calculating the value of $\lambda$ for different random sequences $\omega_{i}$, in the asymptotic limit $n=100000$ bounces, and we obtained in all cases the same Lyapunov exponent.

\subsection{Mixing properties}

Dynamical randomness is characterized by a positive Kolmogorov-Sinai (KS) entropy per unit time $h_{K S} \frac{14}{14}$ In closed systems, it is known that dynamical randomness is a direct consequence of the exponential sensitivity to initial conditions given by a positive Lyapunov exponent. On the other hand, in open dynamical systems with a single Lyapunov exponent $\lambda$, the exponential sensitivity to initial conditions can be related to $h_{K S}$ through the escape rate $\gamma$, by the relation!

$$
\lambda=h_{K S}+\gamma
$$

This formula reveals the fact that in an open dynamical system the exponential sensitivity to initial conditions induces two effects: one is the escape of trajectories out of the neighborhood of 
the unstable reference periodic orbit at an exponential rate $\gamma$, and the other one is a dynamical randomness because of transient chaotic motion near this unstable orbit 15 This dynamical randomness is a measure of the degree of mixing of the system and as mentioned before is quantified by $h_{K S}$. Therefore, for a given $\lambda$, the larger the mixing is, the smaller the escape rate, and vice versa. From Figs $7(b, c)$ it is evident that the Lyapunov exponent and the escape rate have the same smooth dependence on the BS displacement $\Delta$ and that $\gamma \leq \lambda$. We have calculated the difference $\lambda-\gamma>0$ for our system and the result is shown in Figl] (d).

The actual value of $\gamma(\Delta)$ depends, for a fixed value of $\Delta$, on the size of the phase space accessible to the system, $\frac{8}{,}$ that is, it depends on $w_{0}$ and $\theta_{0}$. We verified this behavior by successively decreasing $w_{0}$ and $\theta_{0}$ by factors of 10 (see Table 11), and calculating $\gamma$ for each of these phase space boundaries. It is clear from these results that $\gamma$ increases when the size of phase space decreases; in fact for $w_{0}, \theta_{0} \approx 0$, one should get $\lambda \approx \gamma$ and the cavity mixing property should disappear. It is important to notice that the increment of $\gamma$ with the inverse of the size of the accessible phase space is a general tendency, independent from the arbitrarily chosen boundaries.

\begin{tabular}{|c|c|c|c|c|}
\hline$\left(w_{0}, \theta_{0}\right)$ & $\times 10^{0}$ & $\times 10^{-1}$ & $\times 10^{-2}$ & $\times 10^{-3}$ \\
\hline \hline$\gamma$ & 0.17639 & 0.17596 & 0.19559 & 0.25259 \\
\hline
\end{tabular}

Table 1: Escape rate for different phase space boundaries. As the boundary shrinks $\gamma(\Delta)$ tends to the corresponding value of $\lambda(\Delta)=0.29178 n^{-1}$. In these calculations the displacement of the BS was $\Delta=0.0875 \mathrm{~m}$.

It is important to stress that, although the randomness introduced by the stochastic BS is obviously independent from the cavity characteristics, $\lambda$ and $\gamma$ show a clear dependance on the BS position. When the BS is located at the center of the cavity it is evident for geometrical reasons that the ray splitting mechanism becomes ineffective: $\lambda=0=\gamma$. These results confirm what we have already shown in the SOS (Fig. 4). 


\section{CONCLUSIONS}

We have been able to characterize the ray dynamics of our optical cavity with ray splitting by using standard techniques in nonlinear dynamics. In particular we have found, both through the SOS and the exit basin diagrams, that the stochastic ray splitting mechanism destroys the regular motion of rays in the globally stable cavity. The irregular dynamics introduced by the beam splitter was quantified by calculating the Lyapunov exponent $\lambda$; it grows from zero as the beam splitter is displaced from the center of the cavity. Therefore, the center of the cavity constitutes the only point where the dynamics of the rays is not affected by the stochasticity of the BS. The escape rate $\gamma$ has been calculated and it has revealed a similar dependence with the position of the beam splitter to that of $\lambda$. Furthermore, we have verified that the absolute value of the escape rate tends to that of the Lyapunov exponent as the size of the available phase space goes to zero. This result confirms the fact that the escape rate and therefore the

mixing properties of a map depend sensitively on the choice of the boundary ${ }^{8}$ Because of this dependence we cannot claim that our system is chaotic, despite the positiveness of $\lambda$. However, in a future publication we shall demonstrate that ray chaos can be achieved for the same class of optical cavities when non-paraxial ray dynamics is allowed! 16

This project is part of the program of FOM and is also supported by the EU under the ISTATESIT contract. We thank S. Oemrawsingh for useful contributions to software development.

\section{References}

[1] A. Kohler, G. H. Killesreiter, and R. Blümel, Phys. Rev. E 56, 2691 (1997).

[2] L. Couchman, E. Ott, and T. M. Antonsen, Jr., Phys. Rev. A 46, 6193 (1992).

[3] M. Hentschel and K. Richter, Phys. Rev. E 66, 056207 (2002). 
[4] A. E. Siegman, Lasers (University Science Books, Mill Valley, CA, 1996).

[5] P. Cvitanović et al., Classical and Quantum Chaos (www.nbi.bk/ChaosBook/, 2002).

[6] S. Longhi, Phys. Rev. E 65, 027601 (2002).

[7] E. Ott. Chaos in Dynamical Systems (Cambridge University Press, 2002), 2nd ed.

[8] J. Schneider, T. Tél, and Z. Neufeld, Phys. Rev. E 66, 066218 (2002).

[9] S. Bleher, C. Grebogi, E. Ott, and R. Brown, Phys. Rev. A 38, 930 (1988).

[10] J. Aguirre and M. A. F. Sanjuán, Phys. Rev. E 67, 056201 (2003).

[11] G. Benettin and J. M. Strelcyn, Phys. Rev. A 17, 773 (1978).

[12] H. Fustenberg, Trans. Amer. Math. Soc. 108, 377 (1963).

[13] A. Crisanti, G. Paladin and A. Vulpani, Products of Random Matrices (Springer-Verlag, 1993).

[14] P. Gaspard and G. Nicolis, Phys. Rev. Lett. 65, 1693 (1990).

[15] P. Gaspard, Chaos, Scattering and Statistical Mechanics(Cambridge University Press, 1998), 1st ed.

[16] G. Puentes, A. Aiello, and J. P. Woerdman, in preparation (2003). 


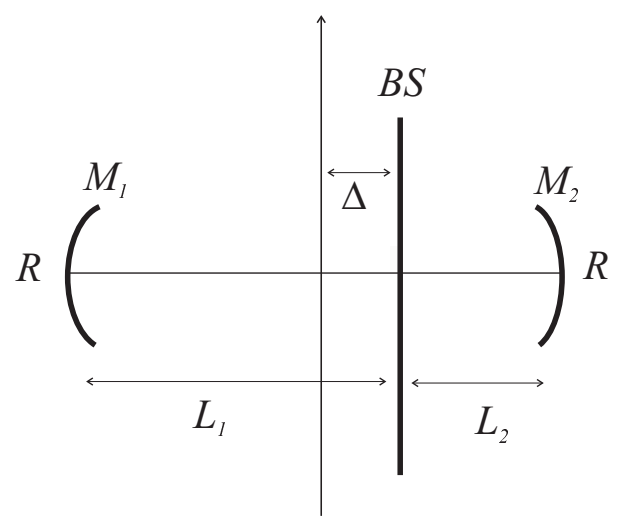

Figure 1: Schematic diagram of the cavity model. Two subcavities of length $L_{1}$ and $L_{2}$ are coupled by a BS. The total cavity is globally stable for $L=L_{1}+L_{2}<2 R$. $\Delta=L_{1}-L / 2$ represents the displacement of the $\mathrm{BS}$ with respect to the center of the cavity.

(a)

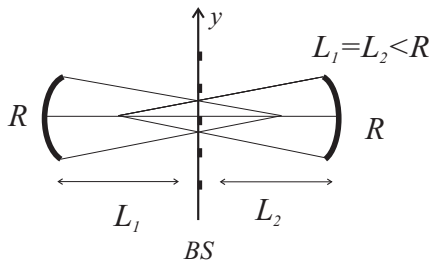

(b)

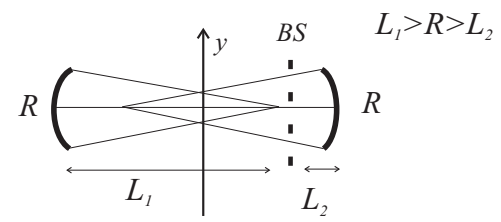

(c)

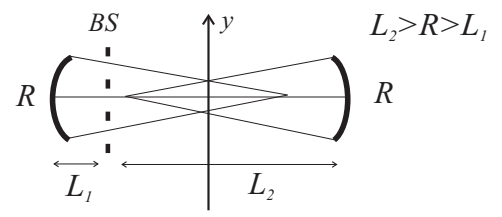

Figure 2: The different positions of the beam splitter determine the nature of the subcavities. In (a) the BS is in the middle, so the 2 subcavities are stable, in (b) the left cavity is unstable and the right one is stable, and (c) the unstable (stable) cavity is on the left (right) (b). 


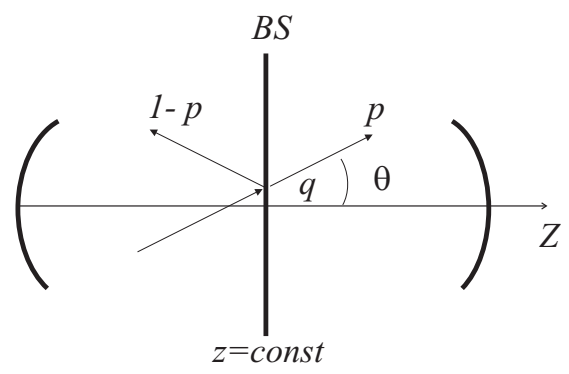

Figure 3: A ray on a reference plane $(z=$ const) perpendicular to the optical axis $(Z)$ is specified by two parameters: the height $q$ above the optical axis and the angle $\theta$ between the direction of propagation and the same axis. When a ray hits the surface of the BS, which we choose to coincide with the reference plane, it can be either reflected or transmitted with equal probability. For a $50 \% / 50 \%$ beam splitter $p=1 / 2$.

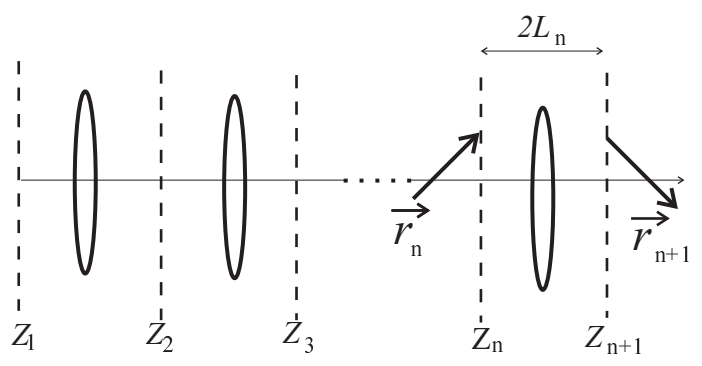

Figure 4: A ray bouncing inside an optical cavity can be represented by a sequence of lenses of focus $f=2 / R$, followed by a free propagation over a distances $L_{n}$. Due to the presence of the BS, the distance $L_{n}$ varies stochastically between $L_{1}$ or $L_{2}$. 
(a)

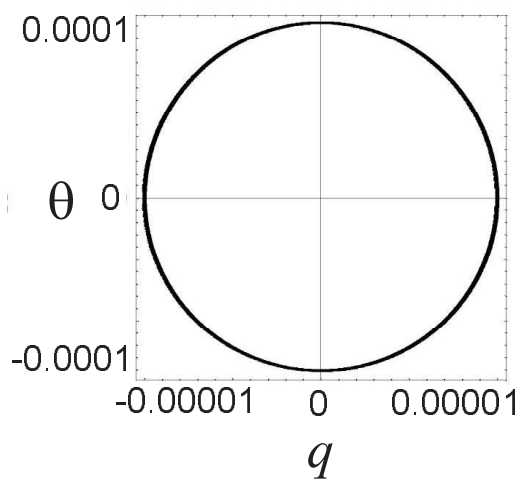

(b)

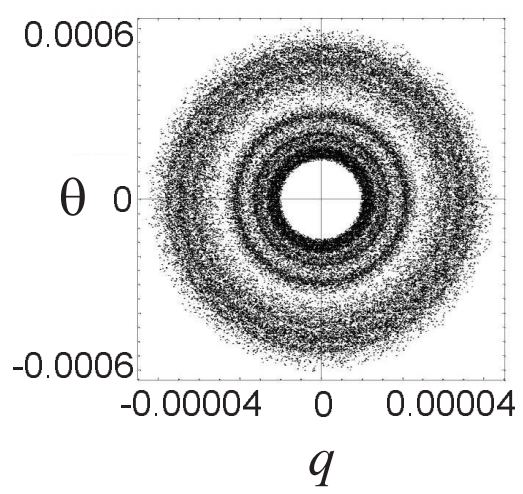

(c)

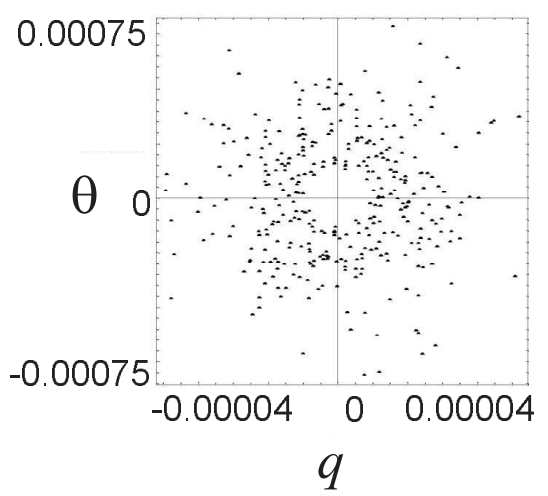

Figure 5: SOS for (a) $\Delta=0$ the ray does not escape, (b) $\Delta=0.001$, the ray escapes after $n=5 \times 10^{4}$ bounces and (c) $\Delta=0.02$, the ray escapes after $n=165$.

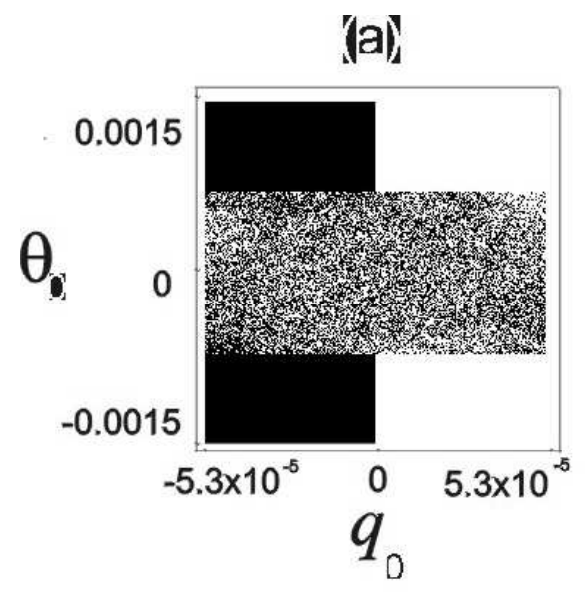

(b)

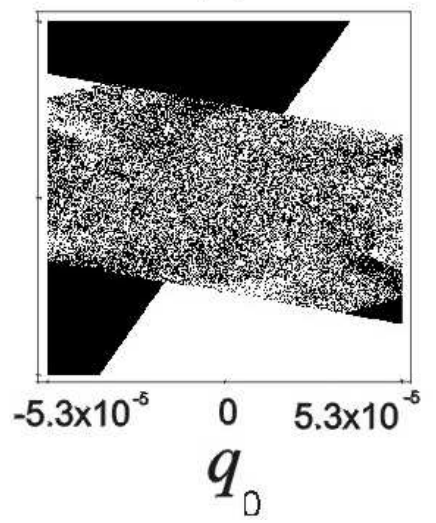

(c)

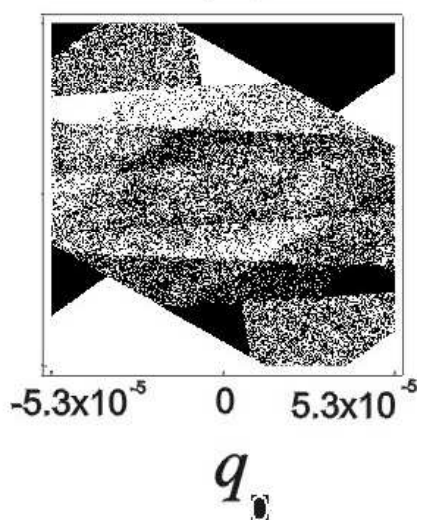

Figure 6: Exit basins for (a) $\Delta=0.025$, (b) $\Delta=0.05$ and (c) $\Delta=0.075$. 

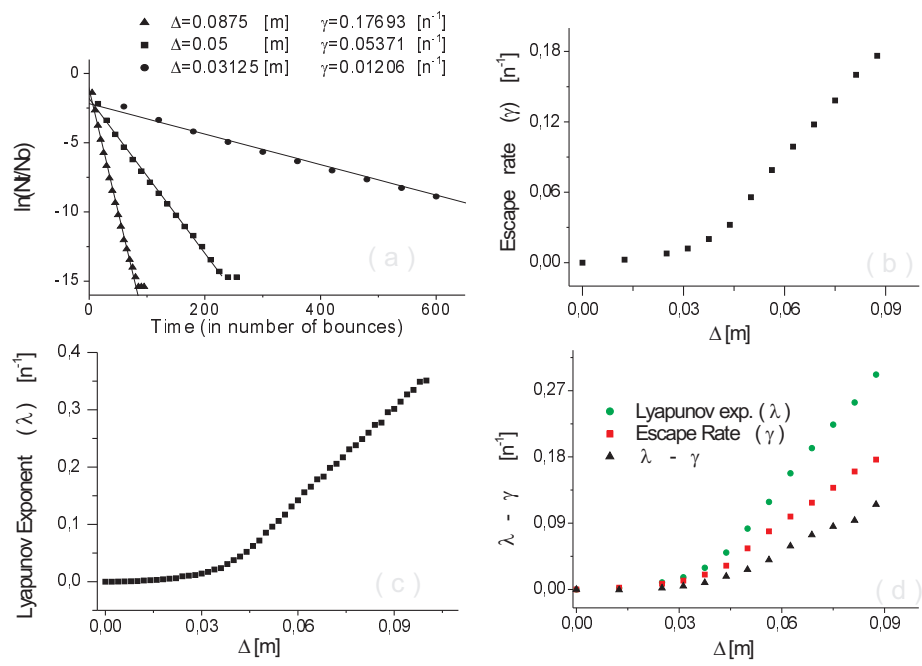

Figure 7: (a) Linear fits used to calculate the escape rate for three different geometrical configurations of the cavity given by $\Delta=0.03125 \mathrm{~m}, \Delta=0.05 \mathrm{~m}$ and $\Delta=0.0875 \mathrm{~m}$. The time is measured in number of bounces $[n]$. The slope $\gamma$ is in units of the inverse of time $\left[n^{-1}\right]$. Fig (b) shows the escape rate $\gamma\left[n^{-1}\right]$ as a function of $\Delta$. Fig. (c) corresponds to different Lyapunov exponents $\lambda\left[n^{-1}\right]$ as the BS moves from the center $\Delta=0$ to the leftmost side of the cavity $\Delta=0.10 \mathrm{~m}$. Fig. (d) shows the difference between $\lambda-\gamma\left[n^{-1}\right]$, which is a positive bounded function. 